\title{
ECZEMA DE CONTACTO ALÉRGICO A ARTROPLASTIAS - UMA RARIDADE OU UMA ENTIDADE SUBDIAGOSTICADA?
}

\author{
Cristina Resende', R. Santos', T. Pereira², C. Araúio', N. Tavares³, C. Brito ${ }^{4}$ \\ 'Interno de Dermatologia e Venereologia /Resident, Dermatology and Venereology. \\ Serviço de Dermatologia e Venereologia, Hospital de Braga \\ ${ }^{2}$ Assistente Hospitalar de Dermatologia e Venereologia/ Consultant Dermatology and Venereology \\ Serviço de Dermatologia e Venereologia do Hospital de Braga \\ ${ }^{3}$ Assistente Hospitalar de Ortopedia/ Consultant Orthopedics. Serviço de Ortopedia do Hospital de Braga \\ ${ }^{4}$ Chefe de Serviço, Diretora do Serviço de Dermatologia e Venereologia, Consultant Chief, Head of Department of \\ Dermatology and Venereology \\ Serviço de Dermatologia e Venereologia do Hospital de Braga, Portugal
}

RESUMO - Introdução: O eczema de contacto alérgico aos constituintes das próteses ocorre em 1-5\% dos doentes e pode ser a causa de falência das artroplastias. Os metais sensibilizantes mais comuns são o níquel, o cobalto e o crómio. Métodos: Estudo retrospectivo dos doentes com artroplastias, submetidos a testes epicutâneos nos últimos 2 anos, com suspeita clinica de eczema de contacto alérgico. Efectuaram-se testes com a bateria padrão do Grupo Português de Estudo das Dermites de Contacto e as baterias complementares de metais, acrilatos/metacrilatos e antibióticos tópicos. Resultados: Foram testados 14 doentes: 10 submetidos a artroplastias do joelho, 3 da anca e 1 do tornozelo. A média de idades foi de 61,6 anos, sendo que 12 eram do sexo feminino. Todos os doentes apresentavam limitação funcional das articulações. A sintomatologia apareceu em média 14,4 meses após a cirurgia. Nove doentes obtiveram positividade ao níquel, 5 ao cobalto, 1 ao crómio, 1 à gentamicina e neomicina. Nesses casos, optou-se pela cirurgia de revisão com substituição por componentes em oxinium e/ou titânio, com melhoria dos sinais inflamatórios. Discussão: Esta revisão mostrou a importância da realização de testes epicutâneos nos doentes com sinais clínicos de falência das artroplastias. Observou-se melhoria das queixas e dos sinais inflamatórios em todos os doentes com sensibilização aos constituintes das artroplastias após a sua substituição. Permanece controverso se os doentes com suspeita de eczema de contacto alérgico a artroplastias deverão ser testados apenas com os seus constituintes ou com uma bateria alargada de alergénios.

PALAVRAS-CHAVE - Artroplastia de substituição; Dermatite alérgica de contacto; Testes epicutâneos.

\section{ALLERGIC CONTACT DERMATITIS TO ARTHROPLASTIES - A RARITY OR A SUBDIAGNOSED ENTITY?}

ABSTRACT - Introduction: Allergic contact dermatitis to the constituents of the prosthesis occurs in 5\% of patients and may be the etiology of the failure of arthroplasty. The most common sensitizer's metals are nickel, cobalt and chromium. The components of the cement have also been implicated as allergens. Methods: A retrospective study of patients with arthroplasties, who performed patch tests, after suspicion of allergic contact dermatitis in the last 2 years was done. We carried out tests with the standard battery of the Portuguese Group for the Study of Contact dermatitis and supplemental batteries, including metals, acrylates/methacrylates and topical antibiotics. Results: Fourteen patients were included: 10 underwent knee arthroplasty, 3 hip arthroplasty and 1 ankle arthroplasty. Patients had limited function of joints. The mean age of patients observed was 61.6 years and 12 patients were females and 2 males. The symptoms appeared on average 14.4 months after surgery. Nine patients had positive reactions to nickel, 5 to cobalt, 1 to chromium, 1 to gentamicin and neomycin. In these cases we opted for placing the prosthesis revision with oxinium 
and/or titanium, with improvement of symptomatology. Discussion and conclusions: This review showed the importance of performing patch tests in patients with clinical signs of failure of arthroplasty, since patients with sensitization to its constituent improved with the replacement of prosthesis.

KEY WORDS - Arthroplasty, replacement; Dermatitis, allergic contact; Patch tests.
Conflitos de interesse: Os autores declaram não possuir conflitos de interesse.
No conflicts of interest.
Suporte financeiro: $O$ presente trabalho não foi suportado por nenhum subsídio ou bolsa.
No sponsorship or scholarship granted.
Direito à privacidade e consentimento escrito / Privacy policy and informed consent: Os autores declaram que pediram consentimento ao doente para usar as imagens no artigo. The authors declare that the patient gave written informed consent for the use of its photos in this article.

Recebido/Received - Dezembro/December 2014; Aceite/Accepted - Janeiro/January 2015

\section{Correspondência: \\ Dr. ㅁ Cristina Resende}

Serviço de Dermatovenereologia

Hospital de Braga

Sete Fontes - São Victor

4710-243 Braga, Portugal

E-mail: cristinapresende@gmail.com

\section{INTRODUÇÃO}

A utilização de próteses de substituição articular nos países ocidentais tem aumentado nos últimos anos com o envelhecimento da população ${ }^{1,2}$. Actualmente, dermatologistas e ortopedistas questionam frequentemente se o desenvolvimento de eczema de contacto alérgico (ECA) aos constituintes das próteses pode ser um fator explicativo de falência das artroplastias, a par de problemas mecânicos e infeciosos ${ }^{3,4}$.

A hipersensibilidade aos componentes metálicos do material implantado é um efeito secundário possível, embora seja difícil de precisar a sua frequência ${ }^{4,5}$. No passado esta situação era considerada rara $(<1 \%)$, mas atualmente alguns estudos demostram que pode chegar aos $5 \%$ 1,2,4.

As próteses da anca são constituídas por um "back metal", com participação de vários tipos de metal; a cabeça da prótese pode ser de metal ou de cerâmica; - acetábulo pode ser constituído por metal (actualmente raro), polietileno ou cerâmica ${ }^{1,3,4}$. A haste das próteses é sempre de metal. As próteses da anca geralmente são não cimentadas ${ }^{1,3}$.

As próteses do joelho possuem dois componentes (femoral e tibial), constituídos por metal, um "insert" articular e "rótula" constituída por polietileno e são geralmente cimentadas ${ }^{3,4}$.

As reacções cutâneas em doentes com artroplastia do joelho são mais frequentes que nas artroplastias da anca $^{1,2}$. As causas para esta diferença são multifactoriais e podem ser explicadas não só pelos constituintes das próteses, mas sobretudo por factores físicos, biológicos e biomecânicos da articulação, que influenciam a libertação de iões metálicos ${ }^{1,2}$.

As próteses podem conter vários metais, nomeadamente níquel, crómio, cobalto, titânio, vanádio, alumínio, molibdénio, manganésio e nióbio'. Os metais sensibilizantes mais comuns são o níquel, o cobalto e o crómio ${ }^{4}$. As respostas de hipersensibilidade ao titânio e vanádio têm sido raramente descritas ${ }^{4}$.

As próteses originais, de primeira geração (metal-metal) desenvolvidas entre 1960 e 1970 foram associadas a sensibilidade ao cobalto, níquel e crómio, bem como a libertação de iões metálicos para o sangue $(28-46 \%)^{3}$. Nos doentes com falência das artroplastias é muitas vezes necessário fazer uma revisão cirúrgica, com substituição por uma prótese com uma constituição diferente'.

As próteses metal-plástico foram usadas de forma crescente de 1970 a 1990 e têm menor risco de 
induzir sensibilização a metais, uma vez que libertam grandes partículas de polietileno, que impedem a formação de complexos proteína-polímero 5 . As próteses metal-plástico não parecem induzir alergia a metais, mas as partículas de polipropileno podem produzir uma reação de corpo estranho no osso, com consequente insuficiência da articulação ${ }^{3}$. Contudo, estas próteses podem levar a reabsorção óssea peri-protési$\mathrm{ca}$, que condiciona a perda da prótese ${ }^{\top}$. Actualmente, nas próteses metal-plástico, utiliza-se polietileno "altamente reticulado", que é mais denso e liberta menos partículas ${ }^{2,3}$.

O reconhecimento da capacidade de partículas do plástico das próteses plástico-metal produzirem uma reação de corpo estranho nos tecidos à volta das articulações, levou ao desenvolvimento de novos tipos de próteses metal-metal na última década ${ }^{4}$. Estas próteses estão a ser usadas pelos cirurgiões ortopédicos em doentes mais novos que a média habitual e que podem requerer mais cirurgias no futuro ${ }^{3,5}$. No entanto, têm sido levantadas questões da sensibilidade aos metais e insuficiência da prótese, bem como o papel que isto pode ter no desenvolvimento de complicações como os "pseudotumores"4-6.

Para além dos metais, os componentes do cimento ósseo têm sido implicados como potenciais alergénios'. O cimento contém iniciadores e estabilizadores de polimerização, antibióticos e acrilatos, que se degradam em pequenas partículas de metacrilatos ${ }^{1}$. A maioria do cimento ortopédico é constituída por metil metacrilato; $\mathrm{N}, \mathrm{N}$-dimetil-p-tolvidina e peróxido de benzoilo ${ }^{5}$. Regra geral, são adicionados antibióticos ao cimento, mais frequentemente a gentamicina ${ }^{5}$.

Este trabalho teve como objectivo avaliar a sintomatologia e relacioná-la com os testes epicutâneos e bem como avaliar o impacto da realização dos testes epicutâneos no prognóstico dos doentes com sinais clínicos de falência de artroplastias.

\section{MATERIAL E MÉTODOS}

Foi efectuado um estudo retrospectivo dos doentes com artroplastias, com suspeita clinica de ECA, submetidos a testes epicutâneos nos últimos 2 anos (2012 e 2013).

Todos os doentes efectuaram testes epicutâneos com a bateria padrão do Grupo Português de Estudo das Dermites de Contacto e com as baterias complementares de metais, acrilatos/metacrilatos e antibióticos tópicos.

\section{RESULTADOS}

No total, foram observados 14 doentes na consulta de Alergologia cutânea, referenciados pela consulta de Ortopedia para exclusão de ECA a constituintes das próteses. Dos doentes estudados, 10 tinham sido submetidos a artroplastias do joelho, 3 da anca e 1 do tornozelo. A média de idades dos doentes foi de 61,6 anos; 12 doentes eram do sexo feminino e 2 do sexo masculino. Ao exame objectivo, todos os doentes apresentavam eritema, calor e edema periarticular, 5 doentes apresentavam exantema à distância e todos os doentes referiam limitação funcional das neoarticulações. A sintomatologia apareceu em média 14,4 meses após a cirurgia.

Nove doentes obtiveram positividade ao níquel, 5 ao cobalto, 1 ao crómio, 1 à gentamicina e neomicina. Nesses casos, optou-se pela cirurgia de revisão com substituição por componentes em oxinium e/ou titânio, com melhoria clínica e dos sinais inflamatórios em todos os doentes no pós-operatório imediato.

As Fig.s 1 e 2 mostram dois exemplos da consulta de doentes que desenvolveram ECA a artroplastias

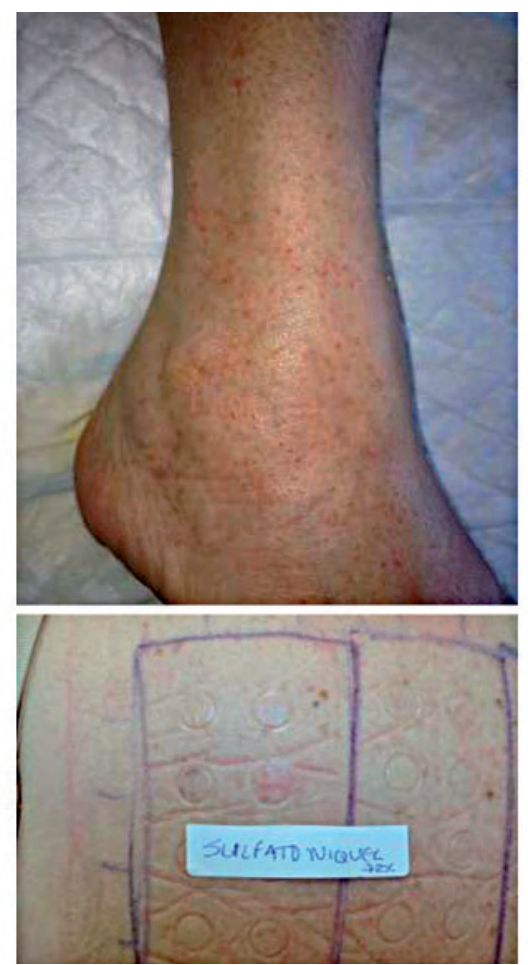

Fig 1 - Lesões de eczema no tornozelo direito, 5 meses após a colocação da prótese; B: Leitura dos testes epicutâneos às 72 horas. 


\section{GPDEC}

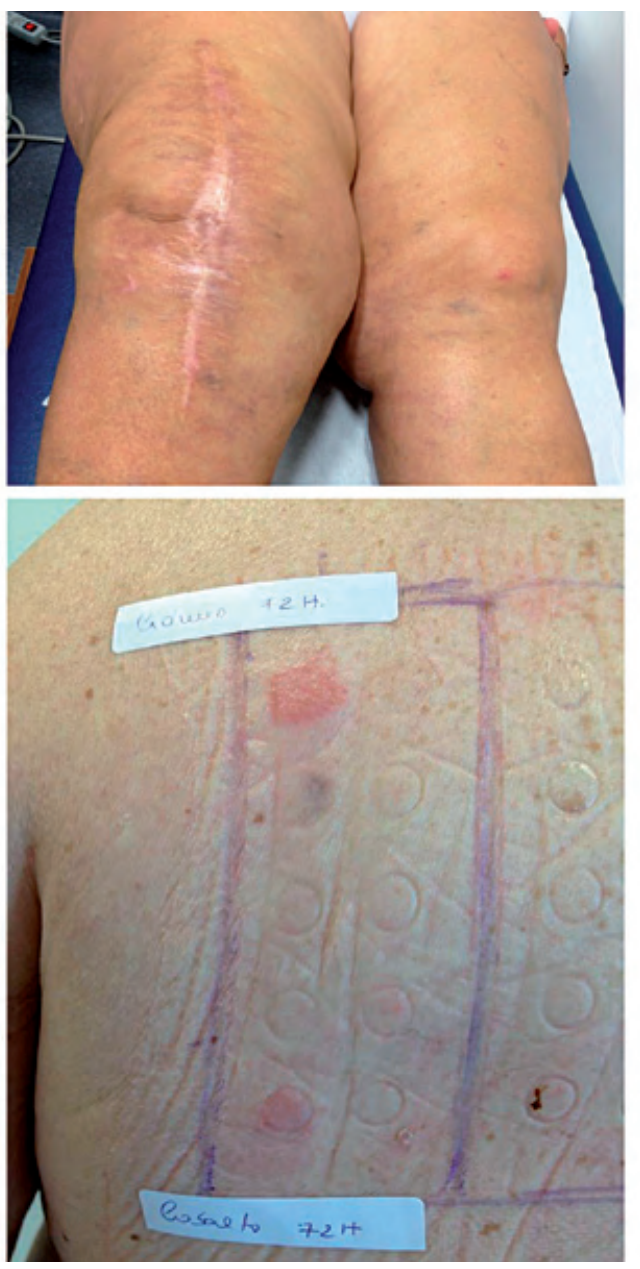

Fig 2 - Edema e rubor do joelho direito, 1 mês após a colocação da prótese; B: Leitura dos testes epicutâneos às 72 horas.

primárias e que tiveram melhoria da sintomatologia após cirurgia de revisão com substituição por componentes em oxinium e/ou titânio.

\section{DISCUSSÃO E CONCLUSÕES}

Os testes epicutâneos são o método de eleição para diagnosticar o $\mathrm{ECA}^{2}$. Uma limitação adicional para estabelecer o papel da sensibilização às próteses ortopédicas é a paucidade de estudos clínicos que mostrem uma clara conexão entre sensibilidade aos metais e a funcionalidade da artroplastia ${ }^{2}$.

No nosso estudo, os testes epicutâneos mostraram positividade com relevância actual aos metais, o que motivou a manutenção de próteses cimentadas. A relevância do metil metacrilato como causa de alergia em doentes com próteses não está bem definida na literatura, apesar de ser um alergénio bem conhecido nos trabalhadores da indústria ortopédica e dentária ${ }^{5}$.

Dos 14 doentes incluídos no nosso estudo, apenas 2 doentes eram do sexo masculino. O número elevado de mulheres pode estar relacionado com o facto da sensibilização aos metais ser superior no sexo feminino.

Esta revisão mostrou a importância da realização de testes epicutâneos nos doentes com sinais clínicos de falência das artroplastias, uma vez que os doentes com sensibilização aos seus constituintes tiveram melhoria das queixas com a sua substituição.

Permanece controverso se os testes epicutâneos devem incluir apenas os componentes das próteses implicadas ou baterias mais alargadas de alergénios ${ }^{7}$. Uma vez que os doentes com insuficiência das artroplastias primárias incluídos no nosso estudo beneficiaram com a substituição da prótese para oxiniun/titânio, é pertinente questionar se se justificará submeter todos os doentes a testes epicutâneos antes da realização da artroplastia primária, com o objectivo de selecionar a prótese mais adequada, mesmo correndo o risco de sensibilização cutânea ${ }^{7}$.

\section{REFERÊNCIAS}

1. Henrique M. Reacções alérgicas a implantes ortopédicos. Bol Inform GPEDC. SPDV. 2012; 70: 7-12.

2. Granchi D, Cenni E, Tigani D, Trisolino G, Baldini N, Giunti A. Sensitivity to implant materials in patients with total knee arthroplasties. Biomaterials. 2008;29(10): 1494-500.

3. Gawkrodger DJ. Metal sensitivities and orthopaedic implants revisited: the potential for metal allergy with the new metal-on-metal joint prostheses. $\mathrm{Br} J$ Dermatol. 2003; 148(6):1089-93.

4. Cousen PJ, Gawkrodger DJ. Metal allergy and second-generation metal-on-metal arthroplasties. Contact Dermatitis. 2012; 66(2):55-62.

5. Basko-Plluska JL, Thyssen JP, Schalock PC. Cutaneous and systemic hypersensitivity reactions to metallic implants. Dermatitis. 2011; 22(2):65-79.

6. Hallab N, Merritt K, Jacobs JJ. Metal sensitivity in patients with orthopaedic implants. J Bone Joint Surg Am. 2001; 83:428-36.

7. Sckalock PC, Menné T, Johansen JD, Taylor JS, Maibach HI, Lidén C, et al. Hypersensitivity reactions to metallic implants-diagnostic algorithm and suggested patch test series for clinical use. Contact Dermatitis, $2012 ; 66$ (1): 4-19. 